\title{
Avaliação acerca do conhecimento sobre a Dengue em jovens em idade escolar
}

\section{Evaluation about Dengue knowledge of children at school age}

Brenda da Silva $1,2,3$, Fernando Garcez Porto ${ }^{4}$, Amanda Marchionatti ${ }^{1}$, Ronaldo dos Santos Machado ${ }^{1}$, Stêfani de Moraes ${ }^{1}$ Julio Candido Schimidt ${ }^{1}$, Barbara Oliveira' ${ }^{1}$, Natalia Helena Mendes ${ }^{5}$, Milene Almeida Ribas ${ }^{6}$, Themis Goretti Moreira Leal de Carvalho ${ }^{1,7}$

${ }^{1}$ Centro de Ciências da Saúde e Agrárias, Universidade de Cruz Alta (UNICRUZ) - Cruz Alta (RS), Brasil. 2Programa de Pós-graduação em Atenção Integral à Sáude, UNICRUZ - Cruz Alta (RS), Brasil.

${ }^{3}$ Universidade do Noroeste do Estado do Rio Grande do Sul (UNIJUí) - ljuí (RS), Brasil.

4Programa de Pós-graduação em Cardiologia, Instituto do Coração da Faculdade de Medicina da USP (FMUSP) - São Paulo (SP), Brasil.

Curso de Psicologia, Complexo de Ensino Superior de Santa Catarina (CESUSC) - Florianópolis (SC), Brasil.

${ }^{6}$ Curso de Fisioterapia, Centro de Ciências da Saúde e Agrárias, UNICRUZ - Cruz Alta (RS), Brasil.

'Núcleo de Pesquisa em Saúde Coletiva, UNICRUZ - Tupanciretã (RS), Brasil.

DOI: https://dx.doi.org/10.7322/abcshs.v44i1.1068

\section{RESUMO}

Introdução: A dengue é uma arbovirose, transmitida para o homem através da picada do mosquito Aedes aegypti, e que acomete grande número de indivíduos no mundo. Nos últimos 50 anos, junto com o aumento da expansão geográfica, a incidência de dengue aumentou aproximadamente 30 vezes. Ao longo dos anos, diversas medidas de combate à dengue foram implementadas, a partir daí, no ano de 2004 foi possível evidenciar que houve redução de $290 \%$ nos casos de dengue em relação ao ano anterior. Objetivo: Descrever o conhecimento de escolares do Rio Grande do Sul sobre o tema dengue. Métodos: Estudo com abordagem quantitativa do tipo descritiva realizado com jovens alunos da rede pública de educação da cidade de Tupãnciretã, por meio de um questionário estruturado. Resultados: Evidenciou-se que a maioria dos alunos tinha conhecimento sobre como a dengue é transmitida, sendo que $35 \%$ acreditavam que a fêmea do mosquito transmite a doença e ainda, 58,8\% afirmaram que conseguem reconhecer o mosquito. Uma parcela dos alunos acredita que o mosquito pica preferencialmente durante o dia, onde a principal forma de transmissão selecionada foi "O mosquito pica uma pessoa doente e transmite a doença à outra pessoa" e os principais sintomas assinalados foram febre, dor de cabeça e cansaço. Conclusão: $O$ estudo demonstrou que boa parte dos jovens possuem conhecimentos sobre a dengue, entretanto, ainda há crianças e adolescentes que não conhecem o assunto, trazendo à tona a importância deste tema nas campanhas de saúde.

\section{ABSTRACT}

Introduction: Dengue is an arbovirus, transmitted to humans through the bite of the Aedes aegypti mosquito, and which affects a large number of individuals in the world. Over the past 50 years, along with increased geographic expansion, the incidence of dengue increased approximately 30 -fold. Over the years, several measures to combat dengue have been implemented and due to this factor, in 2004, there was a $290 \%$ reduction in dengue cases in relation to the previous year. Objective: To describe the knowledge of schoolchildren from Rio Grande do Sul on the subject of dengue. Methods: A quantitative descriptive study carried out with young students of the public education system of the city of Tupãnciretã, through a structured questionnaire. Results: It was evidenced that most of the students had knowledge about how dengue is transmitted, $35 \%$ believed that the mosquito female transmits the disease and $58.8 \%$ claim to be able to recognize the mosquito. A significant portion of the students believe that the mosquito bites preferentially during the day, where the main form of transmission selected was "The mosquito bites a sick person and transmits the disease to the other person" and the main symptoms were fever, headache and tiredness. Conclusion: The study showed that most of the young people have knowledge about dengue, however, there are still children and adolescents who do not know the subject, bringing to the fore the importance of this theme in health campaigns.

Keywords: Dengue; primary prevention; epidemiology.

Palavras-chave: Dengue; prevenção primária; Epidemiologia.

Recebido em: 30/10/2017

Revisado em: 23/06/2018

Aprovado em: 18/09/2018

Autor para correspondência: Themis Goretti Moreira Leal de Carvalho - Universidade de Cruz Alta - Rodovia Municipal Jacob Della Méa, s/n - Parada Benito - CEP: 98020-290 - Cruz Alta (RS), Brasil - E-mail: themiscarvalho@unicruz.edu.br

Conflito de interesses: nada a declarar. 


\section{INTRODUÇÃO}

A dengue é uma arbovirose transmitida para o homem, por meio da picada do mosquito Aedes aegypti, a qual acomete boa parte da população mundial. De maneira específica, nos últimos 50 anos, junto com o aumento da expansão geográfica, a incidência dessa doença aumentou aproximadamente 30 vezes. Além disso, ocorrem anualmente cerca de 390 milhões de infecções por dengue, sendo que 96 milhões dos indivíduos acometidos apresentam manifestações clínicas ${ }^{1,2}$.

Devido a esta alta incidência, no ano de 2005, durante a Assembleia Mundial da Saúde, a dengue foi considerada como uma doença que constitui problema de saúde pública de emergente interesse internacional, devido às implicações para a segurança da saúde pela rápida propagação da epidemia ${ }^{3}$.

Os primeiros relatos de dengue no Brasil ocorreram em meados do século XIX durante o período colonial, advindos de navios negreiros provenientes do continente africano ${ }^{3}$. Antes de 1970 haviam apenas nove países acometidos pela dengue, por outro lado, atualmente a epidemia está presente em mais de 100 países, sendo o Sudeste Asiático e o Pacífico Ocidental às áreas mais afetadas ${ }^{3}$.

$\mathrm{O}$ vírus da dengue é transmitido principalmente pelo mosquito Aedes aegypti, espécie urbana e antropofílica que se reproduz em recipientes artificiais geralmente durante o verão. Essa doença possui características sazonais e no Brasil, os casos de dengue ocorrem principalmente no verão, visto que nesta estação, o mosquito dispõe das condições ideais para reprodução, devido ao aumento da temperatura e frequência de chuvas. Cabe ressaltar que apesar de grande parte desses casos de dengue ocorrer no verão, o Brasil possui os requisitos climáticos necessários para o desenvolvimento e reprodução do mosquito durante todo o ano, e, além disso, o número de casos de dengue vem aumentando ao longo dos últimos anos ${ }^{4,5,6}$. A dengue pode apresentar-se de maneiras distintas sendo classificadas de acordo com as suas manifestações clínicas, com sinais de alerta e grave ${ }^{7}$.

As políticas públicas para o combate da dengue no Brasil vêm evoluindo ao longo dos anos, levando a mudanças significativas. Anteriormente o foco de discussão era voltado à erradicação, porém, a ineficiência em erradicar o mosquito devido a sua rápida proliferação, levou a alterações dessas políticas públicas. Então, visando o controle da doença, o Ministério da Saúde elaborou plano e campanhas para combater o vetor. Ao longo dos anos, diversas medidas de combate à dengue foram implementadas, a partir daí, no ano de 2004 foi possível evidenciar que houve uma redução de $290 \%$ nos casos de dengue em relação ao ano anterior ${ }^{6,8}$.

Essa mudança de foco resultou nas Diretrizes Nacionais para Prevenção e Controle de Epidemias de Dengue, estabelecidas em 2009 pelo Ministério da Saúde em parceria com os Conselhos Nacionais de Secretários Estaduais (CONASS) e Municipais (CONASEMS) de Saúde. Essas diretrizes fornecem suporte para que os planos de prevenção sejam executados de maneira homogênea em todo país 6 .

Outro importante instrumento implantado no Brasil foi o Programa Saúde na Escola (PSE), sendo uma política de interação Saúde e Educação, instituída em 2007. Este programa consiste na união das políticas de saúde e educação com foco nas crianças, adolescentes e adultos da educação pública brasileira, promovendo principalmente saúde e educação integral ${ }^{8}$. A interação entre Escola e Rede Básica de Saúde é a base do PSE, promovendo práticas de prevenção e promoção à saúde, sendo que no ano de 2016 foram lançados vários desafios para mobilização da comunidade escolar contra o Aedes aegypti ${ }^{9}$.

Considerando o aumento no número de casos de dengue, mesmo com a disponibilidade destas políticas públicas de educação, torna-se necessário avaliar o grau de abrangência dos programas de prevenção para a população, em especial à população jovem e em idade escolar, visto que esta é uma fase da vida repleta de experiências que possibilitam sedimentação de conceitos, sendo de grande importância enfatizar a prevenção e promoção de saúde ${ }^{10}$. Deste modo, esta pesquisa se propôs a descrever dados acerca do conhecimento de alunos de uma escola pública do Rio Grande do Sul sobre o tema dengue.

\section{MÉTODOS}

Trata-se de um estudo transversal com abordagem quantitativa do tipo descritiva, realizado com jovens alunos da rede pública de educação da cidade de Tupanciretã, Rio Grande do Sul situada a $408 \mathrm{~km}$ da capital do estado Porto Alegre. Refere-se uma cidade do interior do Rio Grande do Sul com aproximadamente 23.705 habitantes, possuindo uma taxa de escolarização de 11 a 14 anos de idade de $97,9 \%$ com 3.137 alunos matriculados no ensino fundamental e 746 no ensino médio distribuídos em um total de 19 escolas de ensino fundamental e duas escolas de ensino médio. A coleta dos dados foi realizada durante o segundo semestre do ano de 2016.

Para realização da pesquisa houve um contato prévio com a instituição escolar, bem como o encaminhamento de uma solicitação oficial à direção para obtenção da autorização e apresentação do projeto. Após aprovação, foi solicitada a assinatura de um Termo de Autorização por parte da direção, além da assinatura de um Termo de Consentimento Livre e Esclarecido (TCLE) por parte dos estudantes maiores de 18 anos e pelos responsáveis, por parte dos estudantes menores de 18 anos. Foram incluídos todos os estudantes que manifestaram sua intenção de participar do estudo e possuíam consentimento e TCLE assinados por seus responsáveis. Aqueles estudantes que não compareceram no dia da aplicação do questionário ou que não possuíam autorização para participação na pesquisa não foram incluídos no estudo. Esta pesquisa, e o instrumento de coleta de dados seguem as diretrizes 
metodológicas do PSE, além disto, foi enviada ao Comitê de Ética em Pesquisa da Universidade de Cruz Alta e aprovada sob parecer consubstanciado $\mathrm{n}^{\circ}$ 02129512.4.0000.5322.

Como objeto de coleta de dados foi utilizado um questionário estruturado contendo perguntas sobre a etiologia, patogênese e transmissão da dengue, além de dados como: idade, sexo e ano escolar. O questionário é uma maneira simples e rápida de coletar informações sobre determinado tópico dos entrevistados, contudo não testa habilidade do entrevistado. Participaram da pesquisa 1155 alunos da $4^{\text {a }}$ série do Ensino Fundamental até o $3^{\circ}$ Ano do Ensino Médio que concordaram em responder às perguntas propostas e obtiveram Consentimento Livre e Esclarecido de seus responsáveis. A idade, etnia, cor e raça dos estudantes não foram critérios de exclusão nem inclusão para responder ao questionário.

Os dados foram analisados através do software estatístico Statistical Package for the Social Sciences 2.0 e expressos em percentual, para verificar se existe correlação entre o grau de escolaridade e sexo e o conhecimento sobre a dengue e o vetor foi utilizado o Teste de Qui-quadrado, sendo que $\mathrm{p}<0,005$ foi considerado significativo.

\section{RESULTADOS}

Participaram do estudo 1155 alunos de escolas públicas, do município de Tupanciretã, noroeste do Estado do Rio Grande do Sul, com idades entre 10 e 34 anos. Destes, 637 (55,2\%) eram do gênero feminino, 493 (42,7\%) do gênero masculino e 25 (2,2\%) não preencheram o campo gênero do questionário. Quanto ao ano escolar dos participantes, a Tabela 1 traz o número de indivíduos por série escolar.

Quando indagados sobre a forma como a dengue é transmitida para o ser humano, $1004(87,5 \%)$ e 137 (7\%) responderam que sabiam e que não sabiam respectivamente, enquanto que oito $(0,7 \%)$ não assinalaram nenhuma resposta para este questionamento. Foi possível verificar que a série escolar influencia no conhecimento sobre a transmissão da dengue $(\mathrm{p}<0,001)$, mas não altera o grau de conhecimento sobre a morfologia do vetor.

Quando a pergunta foi: "O mosquito da dengue que pica o homem é", 402 (35\%) assinalaram fêmea, 147 (12,8\%) assinalaram macho, 329 (28,7\%) assinalaram não saber a resposta e 269 $(23,4 \%)$ não assinalaram resposta. E quando questionados se saberiam reconhecer o mosquito $674(58,8 \%)$ responderam que sim, 458 (39,9\%) responderam que não, $15(1,3 \%)$ não assinalaram resposta.

As principais características descritas pelos participantes foram pintas brancas 465 (40,5\%), pintas pretas 29 (2,5\%), listras brancas e pretas ao longo do corpo $85(7,4 \%)$ e 568 (49,6\%) não responderam. Quando perguntadas se uma pessoa infectada pode passar a doença para outra, $593(51,7 \%)$ responderam que sim,
$536(46,7 \%)$ responderam que não e $18(1,6 \%)$ não responderam ao questionamento.

Ainda, os participantes da pesquisa responderam a uma pergunta sobre quanto tempo vive o mosquito Aedes aegypti, em que $112(9,7 \%)$ relataram que este vive em torno de 15 dias, 50 $(4,3 \%)$ assinalaram 25 dias, $112(9,7 \%)$ de 30 a 45 dias, $59(5,1 \%)$ aproximadamente um ano e $28(2,4 \%)$ não responderam. Sobre a quantidade de ovos que um mosquito Aedes aegypti é capaz de produzir durante a vida, $163(14,1 \%)$ dos participantes relataram que o mosquito é capaz de produzir até 100 ovos durante a vida, $85(7,4 \%)$ até 200 ovos, $56(4,8 \%)$ até 45 ovos, $112(9,7 \%)$ até 1500 ovos, $711(61,6 \%)$ não souberam responder e 28 (2,4\%) não assinalaram resposta. Quanto ao período do dia em que o mosquito costuma picar, $591(51,2)$ referem que o mosquito pica preferencialmente durante o dia, $221(19,1 \%)$ durante a noite, $329(28,5)$ não souberam responder e $14(1,2 \%)$ não assinalaram resposta.

$\mathrm{O}$ questionamento sobre as formas de transmissão pessoa a pessoa da dengue eram de múltipla escolha e foram expressos na Tabela 2.

Quando foram questionados se o mosquito precisaria estar infectado para transmitir a dengue $818(70,8 \%)$ responderam que sim, 137 (11,9\%) responderam que não, 183 (15,8\%) não

Tabela 1: Número de participantes do estudo por série escolar.

\begin{tabular}{|l|c|c|}
\hline Nível & Ano & $\mathbf{N}(\%)$ \\
\hline \multirow{3}{*}{ Ensino fundamental } & $4^{\text {a }}$ série & $1(0,1)$ \\
\hline \multirow{3}{*}{ Ensino médio } & $5^{\text {a }}$ série & $9(0,8)$ \\
\hline & $6^{a}$ série & $173(15,0)$ \\
\hline & $7^{a}$ série & $200(17,3)$ \\
\hline & $8^{a}$ série & $263(22,8)$ \\
\hline $9^{a}$ série & $131(11,3)$ \\
\hline & $1^{\circ}$ ano & $126(10,9)$ \\
\hline & $2^{\circ}$ ano & $164(14,2)$ \\
\hline & $3^{\circ}$ ano & $42(3,6)$ \\
\hline
\end{tabular}

Tabela 2: Sintomatologia da dengue e número de vezes em que foi assinalada pelos participantes. Questão de múltipla escolha.

\begin{tabular}{|l|c|}
\hline Sintoma & $\begin{array}{c}\mathbf{N}^{\mathbf{0}} \text { de vezes que foi } \\
\text { assinalado pelos alunos }\end{array}$ \\
\hline Cansaço & 603 \\
\hline Diarreia & 320 \\
\hline Dor abdominal & 307 \\
\hline Dor de cabeça & 656 \\
\hline Dor muscular & 581 \\
\hline Dor nas articulações & 568 \\
\hline Falta de apetite & 311 \\
\hline Febre & 894 \\
\hline Náuseas & 387 \\
\hline Vermelhidão na pele & 473 \\
\hline
\end{tabular}


souberam responder e $17(1,5 \%)$ não assinalaram resposta para este questionamento.

Quando perguntados se é possível distinguir a picada do mosquito Aedes aegypti da picada de um mosquito qualquer 229 $(19,8 \%)$ responderam que sim, $304(26,3 \%)$ responderam que não, $598(51,8 \%)$ não souberam responder e $24(2,1 \%)$ não responderam esta questão. Para a pergunta: "Sobre a pessoa que é picada por um mosquito da dengue infectado..." 823 (71,3\%) responderam que esta pessoa fica doente, 231 (20\%) fica doente, mas pode não apresentar os sintomas, $85(7,4 \%)$ responderam que esta pessoa não fica doente e $16(1,4 \%)$ não assinalaram resposta.

Para a pergunta: "É possível confundir a dengue com uma gripe forte?”, $725(62,8 \%)$ relataram que é possível, 94 (8,1\%) assinalaram não ser possível, $305(26,4 \%)$ não souberam responder e 31 $(2,7 \%)$ não responderam à pergunta.

O questionamento sobre a sintomatologia do indivíduo doente possibilitava ao aluno marcar múltiplas respostas e deste modo os dados foram expressos na Tabela 3 .

Sobre o tempo, desde o início da infecção até o aparecimento dos sintomas, $487(42,2 \%)$ assinalaram que os sintomas aparecem entre três e 15 dias, 54 (4,7\%) em até um mês, 589 (51\%) não souberam responder e $25(2,2)$ não assinalaram resposta. Quando indagadas sobre quantas vezes uma pessoa pode desenvolver a

Tabela 3: Formas de transmissão da dengue segundo a percepção dos participantes.

\begin{tabular}{|l|c|}
\hline Meio de transmissão & $\begin{array}{c}\mathbf{N}^{\circ} \text { de vezes que foi } \\
\text { assinalado pelos } \\
\text { alunos }\end{array}$ \\
\hline Água contaminada & 253 \\
\hline Alimento contaminado & 107 \\
\hline Objetos pessoais & 176 \\
\hline $\begin{array}{l}\text { O mosquito pica uma pessoa doente e } \\
\text { transmite a doença à outra pessoa }\end{array}$ & 656 \\
\hline
\end{tabular}

dengue, $216(18,7 \%)$ assinalaram uma vez, 96 (8,3\%) duas vezes, $46(4,0 \%)$ três vezes, $43(3,7 \%)$ quatro vezes, $732(63,4 \%)$ não souberam responder e 22 (1,9\%) não responderam.

Como tratamento para a dengue, 459 (39,7\%) assinalaram que acontece através da ingestão grande quantidade de líquido, Tylenol, Dipirona e repouso, $107(9,3 \%)$ relataram que acontece com o uso de antibióticos, $16(1,4 \%)$ acontece com o uso de Aspirina, $555(48,1 \%)$ não souberam responder e $18(1,6 \%)$ não responderam.

A Tabela 4 evidencia os questionamentos sobre alguns mitos e verdades a respeito do mosquito da dengue.

\section{DISCUSSÃO}

Dos 1155 participantes da pesquisa, $75 \%$ tinham até 16 anos de idade e cursavam até a $8^{\text {a }}$ série do ensino fundamental.

A principal forma de transmissão da doença é através do seu vetor, a fêmea do mosquito Aedes aegypti e o ciclo de transmissão se dá através homem-mosquito-homem ${ }^{11}$, neste estudo $87,5 \%$ dos entrevistados relataram saber como ocorre à transmissão da doença e o conhecimento sobre a doença nesse estudo esteve relacionado à série escolar dos participantes. Estes dados corroboram com o descrito por Kanyangarara et al. ${ }^{12}$ que exploraram o grau de conhecimento de moradores de uma área endêmica da África sobre a malária e verificaram que $85 \%$ dos indivíduos foram capazes de associar o vetor à doença.

A dengue possui uma distribuição a nível mundial, sendo que no ano de 2016 foram reportados grandes surtos de dengue. A Região das Américas registrou mais de 2,38 milhões de casos, onde o Brasil contribuiu com pouco menos de 1,5 milhão de casos, incidência aproximadamente três vezes maior que no ano de 2014. Além disso, foram relatados 1032 óbitos por dengue na região ${ }^{3}$.

Tabela 4: Questionamentos sobre a percepção dos participantes frente a hábitos de prevenção da dengue e condições para o desenvolvimento do mosquito.

\begin{tabular}{|c|c|c|c|c|}
\hline Pergunta & $\begin{array}{l}\text { Verdade } \\
\mathrm{N}(\%)\end{array}$ & $\begin{array}{l}\text { Mito } \\
\mathrm{N}(\%)\end{array}$ & $\begin{array}{c}\text { Não tenho Certeza } \\
\mathrm{N}(\%)\end{array}$ & $\begin{array}{l}\text { Não respondeu } \\
\qquad(\%)\end{array}$ \\
\hline $\begin{array}{l}\text { O mosquito da dengue fica onde o homem estiver e } \\
\text { prefere picá-lo a qualquer outra espécie? }\end{array}$ & $177(15,3 \%)$ & $148(13,7 \%)$ & $802(69,4 \%)$ & $18(1,6 \%)$ \\
\hline $\begin{array}{l}\text { A água de piscina é uma ameaça se não estiver } \\
\text { recebendo o tratamento adequado com aplicação de } \\
\text { cloro em quantidade correta. }\end{array}$ & $910(78,8)$ & $50(4,3)$ & $179(15,5)$ & $16(1,4)$ \\
\hline $\begin{array}{l}\text { São condições ideais para o mosquito procriar e agir: a } \\
\text { temperatura que o mosquito gosta é de } 26-28^{\circ} \text {, qualquer } \\
\text { temperatura inferior à } 18^{\circ} \text { o torna inoperante, com } 42^{\circ} \\
\text { ele morre }\end{array}$ & $314(27,2)$ & $116(10)$ & $713(61,7)$ & $12(1,0)$ \\
\hline $\begin{array}{l}\text { Os ovos ressecados do mosquito da dengue também } \\
\text { são perigosos. Eles sobrevivem até um ano, sem água } \\
\text { e se neste período entram em contato com água, o ciclo } \\
\text { evolutivo começa. }\end{array}$ & $527(45,6)$ & $135(11,7)$ & $457(39,6)$ & $36(3,1)$ \\
\hline
\end{tabular}


A partir disso, tem-se a necessidade da disseminação do conhecimento sobre os meios de transmissão da doença.

Além disto, o Aedes aegypti é o vetor responsável pela transmissão de outras doenças virais agudas tais como Zika e chikungunya. A dengue é uma doença endêmica no Brasil, onde circulam quatro sorotipos do vírus da dengue (DENV), causando grandes epidemias em áreas urbanas. Zika e chikungunya foram recentemente introduzidos nas Américas e se espalharam rapidamente no Brasil ${ }^{13,14,15}$.

Quando questionados sobre o gênero do mosquito que pica o homem e transmite a dengue 402 (35\%) assinalaram que este é fêmea, 147 (12,8\%) assinalaram macho. O vírus que causa a dengue é transmitido aos seres humanos através das picadas de mosquitos fêmeas infectadas, sendo que os seres humanos infectados são os principais portadores e reservatórios do vírus, servindo como fonte de infecção para mosquitos não infectados ${ }^{3}$.

As principais características morfológicas descritas, pelos participantes do estudo, sobre o vetor transmissor da dengue foram pintas brancas 465 (40,5\%), sendo que 568 (49,6\%) não responderam este questionamento indicando desconhecimento sobre a morfologia do mosquito. $\mathrm{O}$ Aedes aegypti possui em média $0,5 \mathrm{~cm}$ de comprimento, tem coloração preta com algumas manchas brancas ao longo do corpo. $\mathrm{O}$ mosquito macho alimenta-se principalmente de frutas devido ao seu caráter adocicado. Por outro lado, a fêmea alimenta-se de sangue (principalmente humano), sendo esta a fonte de alimentação essencial para sua produção dos ovos. E devido a esse fator, que a fêmea contaminada transmite o vírus da dengue para o ser humano ${ }^{14}$.

As fêmeas vivem em torno de 30 a 45 dias e durante a vida podem colocar até 1500 ovos. Estes animais voam a uma altura máxima de meio metro do solo, sendo assim costumam picar nas regiões das pernas, tornozelos e pés ${ }^{14}$. Dos participantes do estudo $593(51,7 \%)$ responderam que a principal fonte de contaminação dos mosquitos não contaminados são os homens doentes, corroborando com isto em nosso estudo, $818(70,8 \%)$ responderam que o mosquito deve estar infectado para transmitir a doença. Ao passo que 591 (51,2\%) referiram que o mosquito pica o homem preferencialmente durante o $\mathrm{dia}^{14}$.

O diagnóstico da dengue ocorre principalmente durante a avaliação clínica e laboratorial. Durante a anamnese o objetivo é pesquisar se o indivíduo apresentou febre, alterações gastrointestinais, do estado de consciência e presença de sangramentos. O MS publicou em 2016 um documento denominado "Dengue diagnóstico e manejo clínico adulto e criança" elaborado com o objetivo de tornar pública a conduta para diagnóstico e tratamento de indivíduos acometidos com a doença no Brasil ${ }^{7}$.

A dengue pode apresentar-se de maneiras distintas sendo classificadas de acordo com as suas manifestações clínicas em dengue, dengue com sinais de alerta e dengue grave. $\mathrm{O}$ caso suspeito de dengue é aquele no qual a pessoa que habite ou tenha viajado nos últimos 14 dias para área onde esteja ocorrendo transmissão de dengue ou tenha a presença de Aedes Aegypti, que apresenta febre, usualmente entre dois e sete dias, e apresente duas ou mais das seguintes manifestações: náusea, vômitos, exantema, mialgias, artralgia, cefaleia, dor retroorbital, petéquias ou prova do laço positiva e/ou leucopenia. Além disto, também pode ser considerado caso suspeito toda criança proveniente ou residente em área com transmissão de dengue, com quadro febril agudo, usualmente entre dois a sete dias, e sem foco de infecção aparente ${ }^{7}$.

Enquanto que, dengue com sinais de alarmes são considerados pacientes que no período de defervescência da febre apresentam dor abdominal intensa e contínua, ou dor a palpação do abdômen, vômitos persistentes, acumulo de líquidos (ascites, derrame pleural, pericárdico), sangramento de mucosas, letargia ou irritabilidade, hipotensão postural (lipotímia), hepatomegalia maior do que $2 \mathrm{~cm}$ e/ou aumento progressivo do hematócrito ${ }^{7}$.

Já a dengue grave é caracterizada pela presença de um ou mais dos seguintes resultados: choque devido ao extravasamento grave de plasma evidenciado por taquicardia, extremidades frias e tempo de enchimento capilar igual ou maior a três segundos, pulso débil ou indetectável, pressão diferencial convergente $\leq 20 \mathrm{~mm} \mathrm{Hg}$, hipotensão arterial em fase tardia, acumulação de líquidos com insuficiência respiratória. Sangramento grave, segundo a avaliação do médico (exemplos: hematêmese, melena, metrorragia volumosa, sangramento do sistema nervoso central), comprometimento grave de órgãos tais como: dano hepático importante (AST o ALT>1000), sistema nervoso central (alteração da consciência), coração (miocardite) ou outros órgãos. Além disto, para confirmar um caso suspeito de dengue devem ser feitos testes laboratoriais (sorologia IgM, NS1 teste rápido ou ELISA, isolamento viral, PCR, Imunohistoquímica $)^{7}$.

O conhecimento acerca da doença e de sua transmissão demonstrado entre os participantes do estudo foi elevado, evidenciaram bom conhecimento da doença o que provavelmente está associado aos programas de prevenção e combate ao vetor. Entretanto, ainda existem crianças e adolescentes que não conhecem o assunto, trazendo à tona a importância deste tema nas campanhas de promoção de saúde, que devem ser cada vez mais intensas, a fim de conscientizar os jovens e continuar incentivando a prevenção contra dengue.

A partir do exposto, torna-se claro que a execução de atividades envolvendo educação e saúde acarretam reflexões e discussões entre os jovens, contribuindo para o conhecimento de diversas doenças, além de conscientizá-los da importância da prevenção. 


\section{REFERÊNCIAS}

1. Chaturvedi UC, Nagar R. Dengue and dengue haemorrhagic fever: Indian perspective. J Biosci. 2008;33(4):429-41.

2. Salles TS, Sá-Guimarães TE, Alvarenga ESL, Guimarães-Ribeiro V, Meneses MDF, Castro-Salles PF, et al. History, epidemiology and diagnostics of dengue in the American and Brazilian contexts: a review. Parasit Vectors. 2018;11(1):264.

https://dx.doi.org/10.1186/s13071-018-2830-8

3. World Organization Health (WHO). Dengue and severe dengue. Disponivel em: http://www.who.int/mediacentre/factsheets/fs117/ en/. Acesso em: 01 nov. 2017.

4. Culshaw A, Mongkolsapaya J, Screaton GR. The immunopathology of dengue and Zika virus infections. Curr Opin Immunol. 2017;48:1-6.

https://dx.doi.org/10.1016/j.coi.2017.07.001

5. Brasil. Ministério da Saúde. Sistema de Informação de Agravos de Notificação (SINAN). Disponível em: http://sinan.saude.gov.br/ sinan/login/login.jsf

6. Brasil. Ministério da Saúde. Secretaria de Vigilância em Saúde. Dengue no Brasil. Informe epidemiológico: monitoramento. Disponível em: http://www.dengue.org.br/boletimEpidemiologico n026.pdf. Acesso em: 01 nov. 2017.

7. Brasil. Ministério da Saúde. Secretaria de Vigilância em Saúde. Departamento de Vigilância das Doenças Transmissíveis. Dengue : diagnóstico e manejo clínico : adulto e criança. 5ed. Brasília: Ministério da Saúde, 2016.

8. 8. Brasil. Ministério da Saúde. Ministério da Educação. Guia para a formação de profissionais de saúde e educação: saúde e prevenção nas escolas. Brasília: Ministério da Saúde; UNESCO; UNICEF; UNFPA, 2007.
9. Ministério da Saúde. Secretaria de Atenção à Saúde. Departamento de Atenção Básica. Caderno de anotações relatos de experiências da Semana Saúde na Escola: Contribuições de troca de experiências de ações de identificação e eliminação dos focos do mosquito Aedes aegypti, associadas a atividades de educação em saúde ambiental para a promoção de ambientes saudáveis, que estão sendo desenvolvidas pelo Brasil afora. Brasília: Ministério da Saúde, 2016.

10. Carvalho TGML, Krabbe EC. Ampliando espaços e articulações: o desafio da prevenção as DST, aids e hepatites virais no IEE Professor Annes Dias. Rev Cataventos. 2015;7(1):1-16.

11. Menchaca-Armenta I, Ocampo-Torres M, Hernández-Gómez A, Zamora-Cerritos K. Risk perception and level of knowledge of diseases transmitted by Aedes aegypti. Rev Inst Med Trop. 2018;60(10):e10

http://dx.doi.org/10.1590/s1678-9946201860010

12. Kanyangarara M, Hamapumbu H, Mamini E, Lupiya J, Stevenson JC Mharakurwa S, et al. Malaria knowledge and bed net use in three transmission settings in southern Africa. Malar J. 2018;17(1):41. http://dx.doi.org/10.1186/s12936-018-2178-8

13. Bhatt S, Gething PW, Brady OJ, Messina JP, Farlow AW, Moyses $\mathrm{CL}$, et al. The global distribution and burden of dengue. Nature. 2013:496(7446):504-7. http://dx.doi.org/10.1038/nature12060

14. Zara ALSA, Santos SM, Fernandes-Oliveira ES, Carvalho RG Coelho GE. Estratégias de controle do Aedes aegypti: uma revisão. Epidemiol. Serv Saúde. 2016;25(2):391-404. http://dx.doi.org/10.5123/s1679-49742016000200017

15. Brasil. Monitoramento dos casos de dengue, febre de chikungunya e febre pelo vírus Zika até a Semana Epidemiológica. Bol Epidemiol. 2017;48(29):1-13 